DOI: $10.20472 /$ IAC.2018.035.029

\author{
LUCIA LEPORATTI \\ University of Genoa - Department of Political Science -, Italy \\ ENRICO DI BELLA \\ University of Genoa - Department of Economics, Italy \\ LUCA GANDULLIA \\ University of Genoa - Department of Political Science -, Italy \\ WALTER LOCATELLI \\ Alisa Regione Liguria, Italy \\ MARCELLO MONTEFIORI \\ University of Genoa - Department of Economics, Italy \\ ROBERTA ZANETTI \\ Alisa Regione Liguria, Italy
}

\title{
FREQUENT USE OF EMERGENCY DEPARTMENTS: AN APPLICATION TO THE PAEDIATRIC CONTEXT
}

\begin{abstract}
:
Frequent users of Emergency Departments (EDs) represent a particularly interesting category of users since they account to a small percentage of patients but they affect considerably accesses, overcrowding and the overall costs of ED activities. The literature on the topic is vast and it allows to delineate a profile of frequent users identifying socio-demographic (age, gender, nationality) and clinical (chronic diseases, drugs and alcohol abuse; psychic illnesses) risk factors. However, most of the studies on the topic limit the analysis to one-year period or to a single study site and there is no consensus on the definition of frequent use. Frequent users are generally defined as those patients reporting a number of accesses per year beyond a certain threshold. The selection of the threshold is often based on previous literature or on percentiles but the definitions vary considerably and the choice tends to be subjective.

In this study, the focus will be placed on paediatric patients, with reference to which the contributions in the literature are still very limited. The objective is to identify the most important drivers of ED frequent use in the 19 EDs of Liguria region (Italy) during a three-year period (2013-2015). The dataset contains 287,242 accesses referred to 144,895 under 14 patients and it includes information on patients' characteristics and on their clinical pathway.

To overcome the limitations connected to previous definitions of frequent use, we exploit the availability of data on three years; this allows to define, not only frequent use, but also its duration (i.e. One-shot / Multiple shot frequent use) and intensity (Normal, High, Very high). By the use of logit and multinomial logit regressions we identify a set of risk factors associated to frequent use and to the different forms of frequent use. Results show that even if frequent users represent a small share of patients (9\%) they contribute to roughly $25 \%$ of accesses. Chronic conditions are the most relevant determinants of frequent use (particularly mental disorders, diseases of the respiratory system) but also foreign nationality turns out to be an important predictor. Differences emerge in the impact of regressors on the different forms of frequent use defined according to its duration and intensity.
\end{abstract}


The study represents an important tool to support policy-making and to discriminate between the potentially preventable frequent use (i.e. inappropriate use) and that associated to complex medical conditions, such as chronic conditions.

\section{Keywords:}

Frequent use, Emergency Departments, Risk factors, paediatric patients

JEL Classification: 110, C50, I18 